\title{
Cuentas ornamentales en un contexto de cazadores-recolectores de la provincia de Córdoba: análisis mineralógico y microestructural de la concha de "Borus"
}

06

Gabriella M. Boretto*, Sandra Gordillo*, Andrés Izeta**, Fernando Colombo", Marisa Martinelli" " y Roxana Cattáneo**:

Recibido:

11 de marzo de 2017

Aceptado:

11 de julio de 2017

\begin{abstract}
Resumen
El objetivo de este trabajo es analizar la composición mineralógica y la microestructura del gasterópodo "Borus" (Megalobulimus lorentzianus) que habita en la provincia de Córdoba y compararla con una cuenta ornamental procedente de un sitio arqueológico de cazadores-recolectores del noroeste de la misma provincia y de esta manera contar con nuevas herramientas para identificar las materias primas utilizadas en su fabricación. El análisis de difracción de rayos X en base a un ejemplar actual indicó que la concha de "Borus" está compuesta en su totalidad de aragonita, polimorfo de carbonato de calcio $\left(\mathrm{CaCO}_{3}\right)$ que cristaliza en el sistema ortorrómbico. Además, el análisis de su microestructura, a través de microscopía electrónica y láser confocal, mostró una disposición prismática de los cristales. Por otro lado, este último análisis realizado en una cuenta proveniente del sitio arqueológico Alero Deodoro Roca (valle de Ongamira, provincia de Córdoba) también indicó la misma composición mineralógica y disposición prismática, lo que se interpreta como un elemento de afiliación al taxón mencionado. Con la incorporación de nuevos materiales este tipo de análisis podrá, a futuro, mejorar el conocimiento respecto a la especialización en la confección de cuentas y posibles redes de intercambio en la región central de Argentina.
\end{abstract}

Ornamental beads in a hunter-gatherers context from the Córdoba Province: a mineralogical and microstructural analysis of a

"Borus" shell

\section{Palabras Clave}

Megalobulimus lorentzianus Composición mineralógica Microestructura Cazadores-recolectores Ongamira

Córdoba

*Centro de Investigaciones en Ciencias de la Tierra (CICTERRA), Universidad Nacional de Córdoba (UNC) CONICET. Av. Vélez Sarsfield 1611 (CP X5016GCA), Córdoba, Argentina. E-mail: gmboretto@yahoo.com.ar; sandra.gordillo@unc.edu.ar; fcolombo@efn.uncor.edu

** Instituto de Antropología de Córdoba (IDACOR), Facultad de Filosofía y Humanidades, Universidad Nacional de Córdoba (UNC) - CONICET. Av. Hipólito Yrigoyen 174 (CP X50ooJHO), Córdoba, Argentina. E-mail: aizeta@ffyh.unc.edu.ar; roxanacattaneo@gmail.com

*** Laboratorio de Materiales Poliméricos, Instituto Multidisciplinario de Biología Vegetal (IMBIV), Universidad Nacional de Córdoba (UNC) - CONICET. Haya de la Torre y Medina Allende, (CP X50ooHUA), Córdoba,

Argentina. E-mail: mmartinelli@fcq.unc.edu.ar 


\begin{abstract}
Keywords

Megalobulimus lorentzianus Mineralogical composition Microstructure Hunter-gatherers Ongamira Córdoba

In this article, we analyze the mineralogical composition and microstructure of the "Borus" gastropod (Megalobulimus lorentzianus), which is local to the Córdoba Province. The gastropod was compared against a typical ornamental bead from a hunter-gatherers archaeological site located in the northwest region of the same province, with the aim of producing new tools to identify the raw materials used in bead manufacture. An X-ray diffraction analysis of a modern "Borus" shell specimen indicated that from a mineralogical standpoint it was composed entirely of aragonite, a calcium carbonate polymorph $\left(\mathrm{CaCO}_{3}\right)$ that crystallizes in the orthorhombic system. In addition, an analysis of its microstructure using laser electron confocal microscope identified a prismatic microstructure. These same analyses, undertaken on a bead from the archaeological site of Alero Deodoro Roca (Ongamira valley, Córdoba Province) revealed the same mineralogical composition and prismatic arrangement, leading to interpret the bead as having a taxonomic affiliation to "Borus" shell. With the addition of new materials, in the future this type of analysis will provide insights regarding specialization in the elaboration of beads and exchange networks in the central region of Argentina.
\end{abstract}

\section{Introducción}

"Borus" es la antigua denominación, aunque muy utilizada en la literatura, de un grupo de caracoles terrestres de gran tamaño de la familia Megalobulimidae, con amplia distribución en Sudamérica (Bequaert, 1948). Para la provincia de Córdoba, la especie citada corresponde a Megalobulimus lorentzianus (Doering, 1876) según revisiones recientes (Beltramino, 2014). En diferentes contextos arqueológicos de la provincia de Córdoba y desde los primeros trabajos (Frenguelli, 1921; Frenguelli y Aparicio, 1932; González y Crivelli, 1978; Menghin y González, 1954; Nimo, 1946; entre otros), se ha mencionado la presencia de "Borus" por la utilización de su concha con fines ornamentales y para la confección de cuentas para la vestimenta o adornos personales (por ejemplo, collares, brazaletes, etc.).

La utilización de las conchas y valvas de moluscos ha sido muy extendida en otras partes del mundo y se remonta a los albores de la humanidad. Uno de los hallazgos que tendría mayor antigüedad (aproximadamente 76.000 años) corresponde a la Cueva de Blombos, en Sudáfrica, donde se encontraron 41 cuentas realizadas con la concha de un caracol marino identificado como Nassarius kraussianus (Henshilwood, D'Errico, Vanhaeren, Van Niekerk y Jacobs, 2004). En este caso la especie pudo ser identificada en base a la preservación de algunos atributos morfológicos reconocibles de sus conchas, que tienen carácter sistemático. Sin embargo, la situación más frecuente es el hallazgo de cuentas que por su grado de manufactura dificultan la identificación de la especie, lo que lleva a la necesidad de contar con otras herramientas para conocer su identidad taxonómica, como son el análisis mineralógico y el microestructural.

Respecto a la utilización del caracol Megalobulimus spp. o "Borus" ha sido de extensa difusión en el noroeste argentino y en otros países de Sudamérica. Si bien hay registros de su utilización con fines alimenticios (ej. Amazonas), en general su valor estuvo asociado a su concha, ya sea como elemento utilitario, ornamental o simbólico. Por ejemplo, "Borus" fue encontrado en contextos funerarios e incluso fuera de su área de distribución, denotando que se trataba de un elemento valioso, de acceso diferenciado y como parte de redes de intercambio, como en el caso de Caral-Supe, Perú (Shady y Leyva, 2003) o en la región de Atacama, Chile (Téllez, 1997). 
En Argentina, uno de los sitios más antiguos donde fue registrada la presencia de este caracol es en el sitio Inca Cueva 4 (capa 1A, datada en 5100 años cal. AP), donde se encontró una multitud de cuentas aserradas y pulidas, confeccionadas a partir de la columela de Megalobulimus sp. (como Strophocheilus sp.; Aschero, 2007) en un contexto funerario y como parte de un ajuar de un cuerpo momificado de una joven.

En la provincia de Córdoba, si bien las cuentas representan uno de los usos más extendidos de los moluscos según se mencionó anteriormente, para la región de Ongamira se han recuperado solamente tres fragmentos manufacturados a modo de cuentas (Figura 1). Cabe aclarar que, para esta región del noroeste de Córdoba y en base a estudios recientes que abordan diferentes tipos de análisis centrados en los moluscos recuperados (Boretto et al., 2014; Gordillo, Izeta, Costa, Boretto y Cattáneo, 2014; Izeta et al., 2014) se asignan otros usos, cuya función se discute aún (Gordillo y Boretto, 2016). De los tres elementos recuperados en el sitio Alero Deodoro Roca en Ongamira, dos de ellos (Figura 1, cuenta en posición derecha e izquierda) se atribuyen a "Borus" en base a sus caracteres preservados. Sin embargo, una tercera cuenta (Figura 1, cuenta en posición central) presenta un notable pulimiento, perdiéndose los caracteres externos diagnósticos y haciéndose necesario incorporar otros elementos diagnósticos como el análisis mineralógico y microestructural.

La utilización de conchas y valvas de moluscos para la confección de cuentas $\mathrm{u}$ otros elementos se vincula a sus excepcionales cualidades mecánicas producto de la biomineralización, que se define como el mecanismo por el cual los organismos vivos forman minerales integrados a una fase orgánica, siendo los principales el carbonato de calcio, el fosfato cálcico, sílice y varios óxidos de hierro (Lowenstam y Wiener, 1989). En los moluscos, la biomineralización de carbonato de calcio se da mayoritariamente en forma de aragonita o calcita, aunque hay otras formas minoritarias como la vaterita (Nehrke, Poigner, Wilhelms-Dick, Brey y Abele, 2012). La calcita y la aragonita son fases polimórficas del carbonato de calcio, dado que tienen la misma composición pero sus estructuras cristalinas son distintas.

Por otro lado, la microestructura de la concha se refiere a la arquitectura de los cristales que la conforman (Carter, 1980), distinguiéndose según la especie una, dos y hasta tres capas diferenciadas por la disposición que adquieren los cristales de carbonato de calcio. Existen diferentes microestructuras, entre ellas: prismática, nacarada, lamelar, laminar entrecruzada, homogénea, lo que varía con los grupos taxonómicos (Carter, 1980, 1990; Taylor, Kennedy y Hall, 1969).

Por lo tanto, teniendo en cuenta la amplia utilización de "Borus" en diferentes contextos arqueológicos, el objetivo de este trabajo es analizar la composición mineralógica y la microestructura de la especie local (Megalobulimus lorentzianus) y compararlas con una cuenta ornamental tipo procedente de un sitio arqueológico de cazadores-recolectores del noroeste provincial, a los fines de contar con nuevas herramientas para identificar las materias primas utilizadas.

\section{Materiales y métodos}

Para la determinación de la composición mineralógica se utilizó un ejemplar actual de Megalobulimus lorentzianus de la colección del Museo de Antropología de la Facultad de Filosofía y Humanidades - Universidad Nacional de Córdoba, procedente de las Sierras de Córdoba (Figura 2). Primeramente, se obtuvieron fragmentos de forma manual y luego se realizó un pretratamiento que consistió en un cepillado y lavado a través de ultrasonido durante 10 minutos para la extracción de todo tipo de sedimento que pudiera haber quedado adherido. Posteriormente el material se pulverizó utilizando 


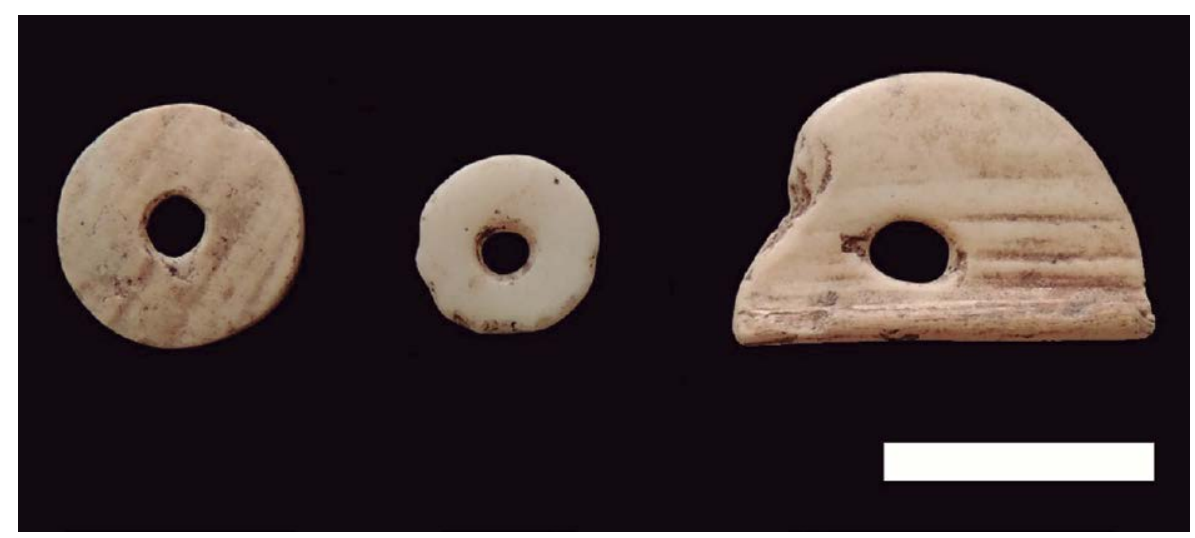

Figura 1. Cuentas recuperadas en el sitio Alero Deodoro Roca, valle de Ongamira (provincia de Córdoba). Se estima una edad de 3000 años AP (Cattáneo et al., 2013; lzeta et al., 2014). Escala gráfica $=10 \mathrm{~mm}$.
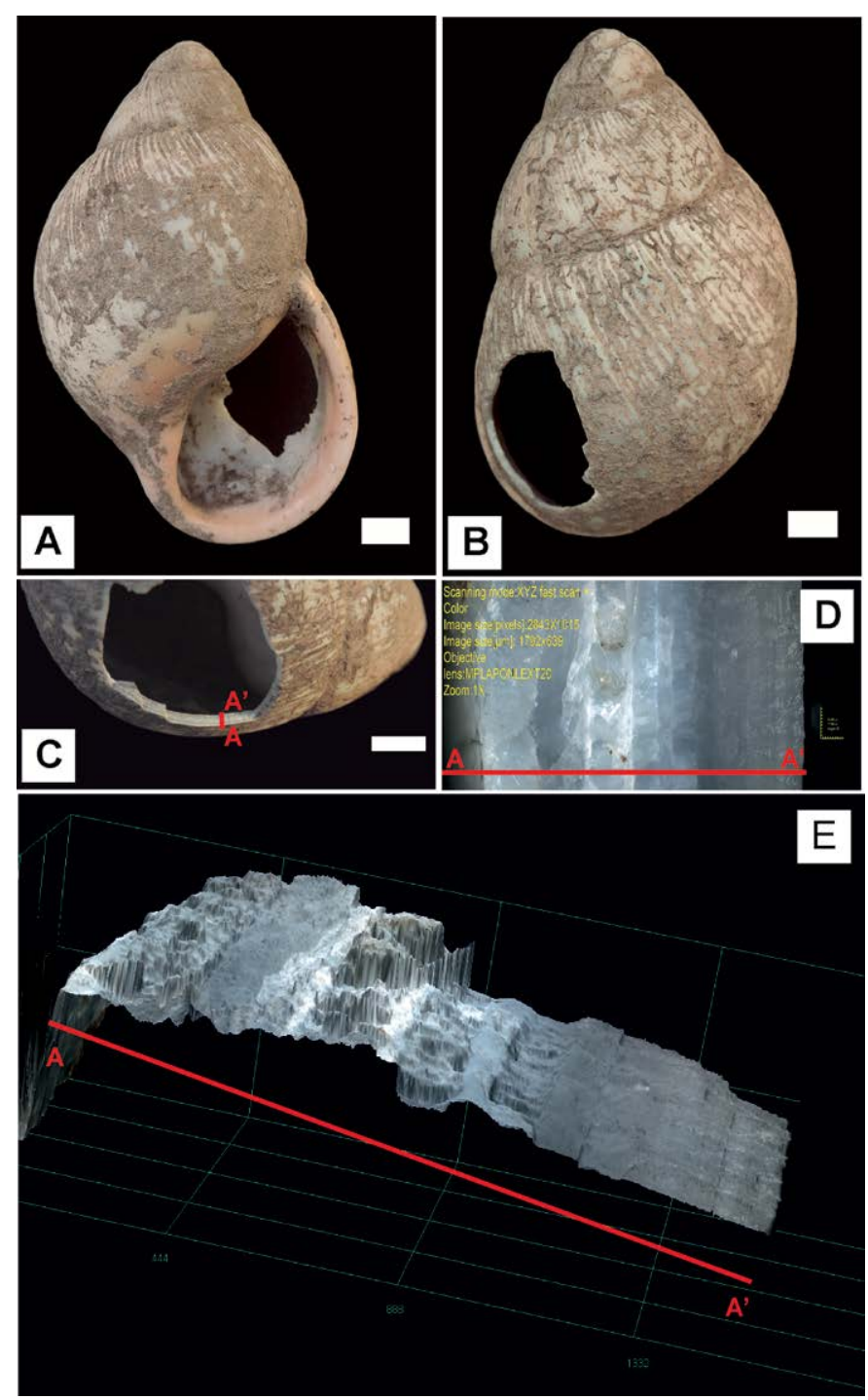

Figura 2. Análisis microestructural de un ejemplar de "Borus" (Megalobulimus lorentzianus). A y B) Vistas externas del ejemplar; $C$ ) Detalle de sector donde se visualiza el área $\left(A-A^{\prime}\right)$ analizada en el microscopio láser confocal. Escala gráfica $=10 \mathrm{~mm} ; \mathrm{D}$ ) Vista de la microestructura con magnificación de 428X; E) Vista de la microestructura con magnificación de 1000X. 
un mortero de ágata. A continuación, para los estudios de difracción de rayos X (DRX) se utilizó un equipo Philips PANalytical X'Pert Pro (INFICQ, CONICET-Universidad Nacional de Córdoba) con radiación Cu Ka obtenida a $40 \mathrm{kV}$ y $40 \mathrm{~mA}$, entre 5 y 100 $(2 \theta)$, con un paso de $0,02^{\circ}(2 \theta)$ y 10 s de conteo por paso.

En el caso de la cuenta arqueológica, con el fin de conocer su composición mineralógica pero sin tener que alterarla ni romperla y así preservarla, se utilizó un método de detección alternativo a la DRX, empleándose espectroscopía FT-IR. Esta técnica no destructiva consiste en analizar la banda infrarroja del espectro electromagnético para identificar la composición de una muestra. El material sólido a estudiar se monta en una platina y un rayo de luz infrarroja lo atraviesa sin necesidad de modificarlo, obteniéndose una curva del espectro infrarrojo. A los fines de asociar el espectrograma obtenido de la cuenta con las especies minerales de carbonato de calcio más distribuidas, es decir aragonita y calcita, también se realizaron espectrografías de ambos minerales que sirvieron de patrón (Adler y Kerr, 1962; Singh, Kumar, Waghmare, Sabale, 2016; Weir y Lippincott, 1961). Los espectros fueron obtenidos usando un equipo Thermo Scientific Nicolet iN10 Infrared Microscope con detector MCT-LN2 entre 650 y $4.000 \mathrm{~cm}-1$ con una resolución de 4 cm-1 (LaMaP, Departamento de Química, Facultad de Ciencias Químicas, Universidad Nacional de Córdoba, CONICET). Pequeñas fracciones de cada muestra fueron colocadas sobre discos de $\mathrm{KBr}$ (bromuro de potasio) dado que el detector MCT permite registrar tamaños de muestras de 10 micrones o menos.

Para el análisis de la microestructura se utilizaron fragmentos del mismo espécimen, pero además se incorporó una cuenta ornamental procedente del sitio arqueológico Alero Deodoro Roca, en el valle de Ongamira, con una edad estimada en 3000 años cal. AP (Cattáneo, Izeta y Takigami, 2013). Este análisis se llevó a cabo en el Laboratorio de Microscopía Electrónica y Análisis por RX (LAMARX, FaMAF, Universidad Nacional de Córdoba, CONICET). Para ello, tanto los fragmentos de concha seleccionados como la cuenta ornamental fueron montados en la platina del microscopio láser confocal Olympus Lext OLS4000, obteniéndose imágenes del perfil de la pared de la concha y de la cuenta con diferentes aumentos (108X, 428X, 1000X) a los fines de caracterizar las propiedades intrínsecas de este taxón. Cabe aclarar que el uso de láser con el Lext es una técnica no destructiva.

\section{Resultados}

El análisis de difracción de Rayos $\mathrm{X}$ indicó que la composición mineralógica de la concha de Megalobulimus lorentzianus está constituida en su totalidad de aragonita, polimorfo de carbonato de calcio $\left(\mathrm{CaCO}_{3}\right)$ que cristaliza en el sistema ortorrómbico.

La concha de Megalobulimus lorentzianus presenta un gran desarrollo del grosor de la valva, característica que permite que el exoesqueleto sea resistente y durable. El valor de este parámetro medido a través de calibre electrónico fue de 1,7 $\mathrm{mm}$ en el sector más engrosado (Figura 2C, perfil A-A'), siendo el valor medio de 1,51 mm y el valor mínimo de $1,15 \mathrm{~mm}$ próximo a los bordes. La microestructura se caracteriza por un acentuado crecimiento prismático, siendo este su rasgo distintivo (Figura $2 \mathrm{D}$ y E).

Respecto a la cuenta analizada (Figura 3), se identifica un desarrollo anisótropo de la microestructura, en la cual los cristales de carbonato tienden a seguir una dirección, siendo compleja su distinción específica dado el grado de pulimiento que presenta, aunque al utilizar la máxima resolución del microscopio láser se reconoce el desarrollo de la microestructura prismática que se correspondería con la superficie externa de la concha, en coincidencia con la especie analizada. 


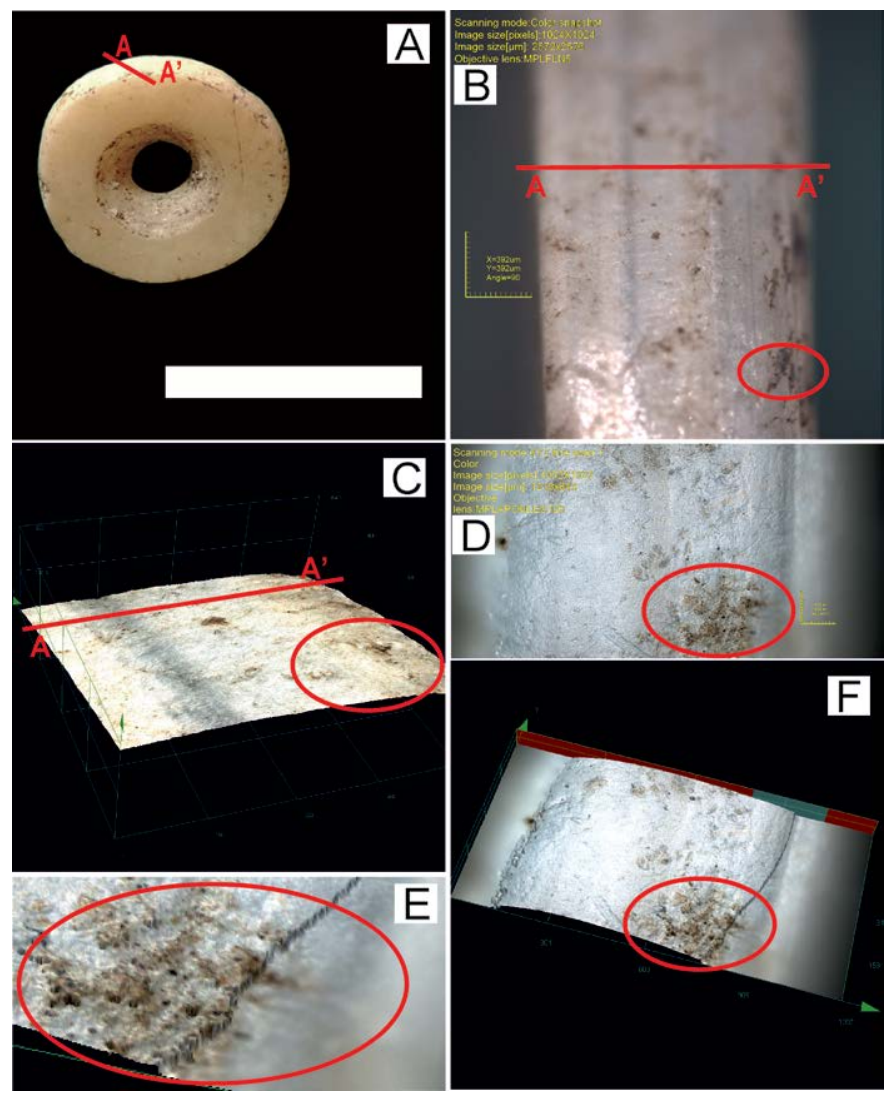

Figura 3. A) Cuenta ornamental hallada en contexto arqueológico en el sitio Alero Deodoro Roca, valle de Ongamira, perfil A-A' es el sector estudiado. Escala gráfica $=10 \mathrm{~mm}$; $B$ ) Vista del perfil A-A' para análisis microestructural de la cuenta con magnificencia de $100 X$ mediante microscopio láser confocal; C) Vista en $3 D$ de la microestructura con magnificencia de 400X; D) Vista en $2 D$ de la microestructura con magnificencia de $428 X ; E$ y F) Detalle del desarrollo de la microestructura columnar observada en la cuenta en vista $3 D$ con magnificencia de 1000X.

El análisis de la composición mineralógica de la cuenta indica que su composición es aragonítica, coincidiendo la frecuencia del espectro infrarrojo de la misma con el espectograma de la aragonita patrón (Figura 4).

\section{Discusión}

Las conchas de los caracoles se forman a través de un proceso de biomineralización que consta de una fase orgánica y otra inorgánica, dando por resultado una estructura conformada en capas. La capa más externa, no considerada en este trabajo, corresponde al perióstraco, que precede a la depositación mineral y es esencialmente orgánica, proteica, aunque en parte mineralizada (de Paula y Silveira, 2009). Esta porción normalmente no se preserva e incluso se va perdiendo cuando el animal está aún con vida. Sin embargo, ejerce un importante control en el proceso de construcción de la concha (de Paula y Silveira, 2009; Galindo Ávila, 2013; Pavat et al., 2012). La fase inorgánica o mineral es la que habitualmente queda preservada en el registro arqueológico y paleontológico y en los moluscos se compone de carbonato de calcio $\left(\mathrm{CaCO}_{3}\right)$ que varía en su forma de cristalización.

En esta contribución se demostró a través de la difracción por rayos X que la fase mineral de la concha de Megalobulimus lorentzianus está formada 100\% por 


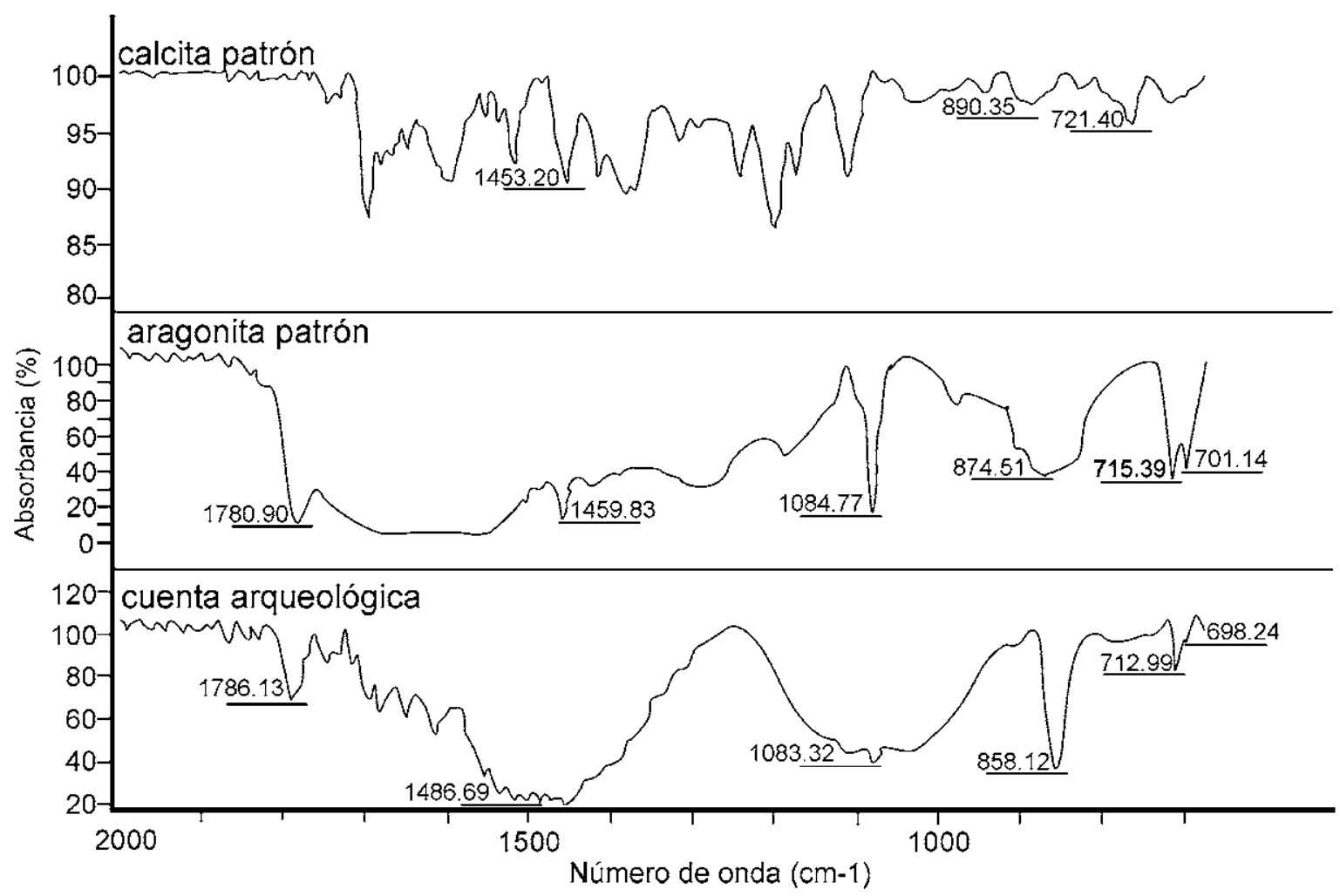

Figura 4. Espectrogramas en la banda infrarroja de las estructuras de calcita patrón, aragonita patrón y cuenta arqueológica obtenido mediante espectroscopía FT-IR.

aragonita, y, a partir de la microscopía electrónica láser confocal, que la aragonita cristalizó con un marcado crecimiento prismático, con cristales paralelos entre sí y perpendiculares al plano de superficie. Este patrón de estructura prismática se observa en otras especies de moluscos, ya sean aragoníticas o calcíticas y generalmente es más prominente en los bivalvos (Carter, 1980; de Paula y Silveira, 2009). Como se ha mencionado anteriormente, la biomineralización hace que las conchas y también los elementos obtenidos a partir de ellas como las cuentas, tengan excepcionales cualidades mecánicas, lo que seguramente fue una de las razones de su utilización como materia prima para elaborar diferentes elementos. Asimismo, el análisis mineralógico a través espectroscopía FT-IR realizado en la cuenta indicó que el espectrograma obtenido coincide con el de patrón de aragonita. Los resultados de ambos análisis, es decir el microestructural y el mineralógico, coinciden tanto para la concha de Megalobulimus lorentzianus como en la cuenta arqueológica recuperada, por lo tanto se establece que la concha de este gasterópodo ha sido la materia prima utilizada para la realización de este tipo de ornamentos.

Respecto a la utilización de otras especies de moluscos terrestres nativos para la elaboración de cuentas en la provincia de Córdoba, la única otra especie que podría haberse utilizado, teniendo en cuenta su tamaño mayor a una cuenta tipo, es Austroborus cordillerae, especie emparentada con Megalobulimus lorentzianus. Sin embargo, A. cordillerae es una especie endémica del noroeste de Córdoba, sumamente escasa (Gordillo et al., 2014), por lo que una especialización en la fabricación de cuentas a partir de la misma no habría sido factible, salvo para uso diferencial y muy exclusivo y/o simbólico. Otras especies nativas terrestres que aparecen enteras en yacimientos arqueológicos de Córdoba, como por ejemplo en Ongamira (Gordillo y Boretto, 2016; 
Izeta et al., 2014), incluyen a Spixia spp., Plagiodontes spp. y Epiphragmophora spp., pero dado su pequeño tamaño y/o delgadez, no se condicen con las materias primas de una cuenta tipo. Además, cabe añadir que la especie Epiphragmophora spp. se caracteriza por poseer microestructura laminar entrecruzada en su capa más externa (Boretto, Izeta, Cattáneo y Gordillo, 2015), cuyo rasgo distintivo no se asocia a la microestructura de la cuenta observada.

Sin embargo, otras especies no nativas de la región podrían haber arribado a través de redes de intercambio. Al respecto, se ha constatado para Córdoba la presencia de especies de agua dulce como las almejas náyade que viven en el NE de Argentina en el área de la cuenca del Paraná y Anodontites trapesialis y Diplodon parallelopipedon hallados en el noreste de Córdoba (Fabra y Gordillo, 2014; Fabra, Gordillo y Piovano, 2012), además de moluscos marinos como Urosalpnix sp. (Serrano, 1945) entre otros. Respecto a Anodontites trapesialis, el análisis del patrón microestructural realizado por Callil y Mansur (2005) también fue utilizado como elemento diagnóstico para corroborar la presencia de esta especie en el contexto arqueológico del área de Mar Chiquita (Fabra et al., 2012).

\section{Comentarios finales}

Este trabajo aporta información conquiliológica sobre la composición mineralógica y microestructural de "Borus" a los fines alcanzar una aproximación a la identidad taxonómica de la especie que aportó la materia prima para la confección de cuentas. Sin embargo, y dada la presencia de otras especies como las almejas náyades, es factible que parte de las cuentas halladas en Córdoba se hayan confeccionado además con otras especies. Futuros trabajos centrados en el análisis mineralógico y microestructural de cuentas de esta misma especie o de otras especies de uso potencial para la elaboración de cuentas de diferentes sitios arqueológicos en la provincia de Córdoba, podrán brindar mayor información sobre la materia prima utilizada y así mejorar el conocimiento respecto a la confección de cuentas y posibles redes de intercambio.

\section{Agradecimientos}

Los autores agradecen a dos revisores anónimos y al comité editorial por los comentarios y sugerencias. Este trabajo se enmarca en el proyecto de la ANPYCT: "Arqueología de grupos cazadores recolectores de las sierras pampeanas australes” (PICT 2011-2122). Se agradece además al Lic. Santiago Druetta por las fotografías tomadas del material de estudio. 


\section{Referencias citadas}

" Adler, H. H. y Kerr, P. F. (1962). Infrared study of aragonite and calcite. The American Mineralogist, 47, 700-717.

"Aschero, C. A. (2007). Iconos, Huancas y Complejidad en la Puna sur argentina. En A. Nielsen, M. C. Rivolta, V. Seldes, M. Vázquez y P. Mercolli (Eds), Producción y Circulación Prehispánicas de Bienes en el Sur Andino (pp.135-165). Colección Historia Social Precolombina 2. Córdoba: Editorial Brujas.

» Beltramino, A. A. (2014). Distribución histórica y área de distribución potencial del megamolusco terrestre Megalobulimus lorentzianus (Doering, 1876) (Gastropoda: Pulmonata) en América del Sur. Boletín de la Asociación Argentina de Malacología, 4(1), 10-13.

» Bequaert, J. C. (1948). Monograph of the family Strophocheilidae, a Neotropical family of terrestrial mollusks. Bulletin of the Museum of Comparative Zoölogy Harvard, 100, 1-210.

» Boretto, G., Izeta, A., Cattáneo, R. y Gordillo, S. (2015). Cuentas ornamentales en contexto arqueológico en la provincial de Córdoba: análisis microestructural de valvas de moluscos para su identificación taxonómica. Trabajo presentado en VI Congreso Argentino de Cuaternario y Geomorfología, Ushuaia, Argentina.

" Boretto, G., Robledo, A., Izeta, A., Baranzelli, M., Gordillo, S. y Cattáneo, R. (2014). Análisis morfométrico de ejemplares actuales y fósiles de Plagiodontes daedaleus (Deshayes 1851) del sitio Alero Deodoro Roca, Sierras Pampeanas de Córdoba, Argentina. En H. Hammond y M. Zubimendi (Eds.), Arqueología y Malacología: Abordajes Metodológicos y Casos de Estudio (pp. 91-104). Buenos Aires: Fundación de Historia Natural Félix de Azara.

»Callil, C. T. y Mansur, M. C. D. (2005). Ultrastructural analysis of the shells of Anodontites trapesialis (Lamarck) and Anodontites elongatus (Swaison) (Mollusca, Bivalvia, Etherioidea) from the Mato Grosso Pantanal Region, Brazil. Revista Brasileira de Zoologia, 22(3), 724-734.

"Carter, J. G. (1980). Environmental and biological controls of bivalve shell mineralogy and microstructure. En D. C. Rhoads y R. A. Lutz (Eds.), Skeletal Growth of Aquatic Organisms: Biological Records of Environmental Change (pp. 203-249). Nueva York: Plenum Press.

"Carter, J. G. (1990). Skeletal Biomineralization: Patterns, Processes and Evolutionary Trends. Nueva York: Van Nostrand Reinhold.

»Cattáneo, G. R., Izeta, A. D. y Takigami, M. (2013). Primeros fechados radiocarbónicos para el Sector B del sitio Alero Deodoro Roca (Ongamira, Córdoba, Argentina). Relaciones de la Sociedad Argentina de Antropología, XXXVIII(2), 559-567.

» de Paula, S. M. y Silveira, M. (2009). Studies on molluscan shells: contributions from microscopic and analytical methods. Micron, 40, 669-69o.

"Doering, D. A. (1876). Apuntes sobre la fauna de moluscos de la República Argentina (Tercera parte). Boletín de la Academia Nacional de Ciencias Exactas en Córdoba, 2, 300339.

» Fabra, M. y Gordillo, S. (2014). Estimaciones acerca del uso de una almeja de agua dulce (Diplodon parallelopipedon) hallada en contexto arqueológico en el Mar del Ansenuza (Córdoba, Argentina). En H. Hammond y M. Zubimendi (Eds.), Arqueología y Malacología: Abordajes Metodológicos y Casos de Estudio (pp. 129.143). Buenos Aires: Fundación de Historia Natural Félix de Azara. 
» Fabra, M., Gordillo, S. y Piovano, E. (2012). Arqueomalacología en las costas de Ansenuza: análisis de una almeja nacarífera (Anodontites trapesialis) hallada en contexto funerario del sitio El Diquecito (Laguna Mar Chiquita, Córdoba). Revista Arqueología, 18, 257-266.

"Frenguelli, J. (1921). Conchas de "Borus" en los paraderos indígenas del río San Roque (Sierra Chica de Córdoba, departamento de Punilla). Boletín de la Academia Nacional de Ciencias de Córdoba, 26, 404-418.

» Frenguelli, J. y De Aparicio, F. (1932). Excursión a la Laguna de Mar Chiquita (provincia de Córdoba). Publicaciones del Museo Antropológico y Etnográfico de la Facultad de Filosofía y Letras, Serie A(II), 121-166.

" Galindo Avila, A. (2013). Efecto de la matriz orgánica de picoroco sobre la reparación de la concha del caracol de tierra Helix aspersa. (Tesis de Pregrado), Universidad de Chile, Chile. Disponible en http://repositorio.uchile.cl/handle/225o/131572 (Acceso: 28 de junio de 2017).

»González, S. y Crivelli, E. (1978). Excavaciones Arqueológicas en el Abrigo los Chelcos. Relaciones de la Sociedad Argentina de Antropología, XXIII, 183-216.

» Gordillo, S. y Boretto, G. (2016). Análisis de conjuntos arqueomalacológicos en el valle de Ongamira. En R. Cattáneo y A. Izeta (Eds.), Arqueología en el Valle de Ongamira (20102015) (pp. 143-162). Córdoba: Universidad Nacional de Córdoba.

» Gordillo, S., Izeta, A., Costa, T., Boretto, G. y Cattáneo, R. (2014). Austroborus cordillerae (Doering 1877) en el Valle de Ongamira: una especie endémica del noroeste de Córdoba en contexto arqueológico de cazadores-recolectores. En H. Hammond y M. Zubimendi (Eds.), Arqueología y Malacología: Abordajes Metodológicos y Casos de Estudio (pp. 119127). Buenos Aires: Fundación de Historia Natural Félix de Azara.

"Henshilwood, C. S., D’Errico, F., Vanhaeren, M., Van Niekerk, K. y Jacobs, Z. (2004). Middle Stone Age shell beads from South Africa. Science, 304(5669), 404.

»Izeta, A., Costa, T., Gordillo, S., Cattáneo, R., Boretto, G. y Robledo, A. (2014). Los gasterópodos del sitio Deodoro Roca, Valle de Ongamira (Córdoba, Argentina). Un análisis preliminar. Revista Chilena de Antropología, 29, 74-80.

» Lowenstam, H. A. y Weiner, S. (1989). On Biomineralization. Oxford: Oxford University Press.

» Menghin, O .F. A. y González, A. R. (1954). Excavaciones arqueológicas en el yacimiento de Ongamira, Córdoba (Rep. Arg.) (Nota preliminar). Notas del Museo de La Plata XVII, Antropología, 67, 213-268.

» Nehrke, G., Poigner, H., Wilhelms-Dick, D., Brey, T. y Abele, D. (2012). Coexistence of three calcium carbonate polymorphs in the shell of the Antarctic clam Laternula elliptica. Geochemistry Geophysics Geosystems, 13, Q05014.

"Nimo, A. (1946). Arqueología de Laguna Honda (Yucat, Provincia de Córdoba). Publicaciones del Instituto de Arqueología, Lingüística y Folklore "Dr. Pablo Cabrera", XVI, 977-1045.

» Pavat, C., Zanella-Cléon, I., Becchi, M., Medakovic, D., Luquet, G., Guichard, N., Alcaraz, G., Dommergues, J., Serpentini, A., Lebel, J. y Marin, F. (2012). The shell matrix of the pulmonate land snail. Helix aspersa maxima. Comparative Biochemistry and Physiology Part B: Biochemistry and Molecular Biology, 161(4), 303-314.

» Serrano, A. (1945). Los Comechingones. Córdoba: Instituto de Arqueología, Lingüística y Folklore de la Universidad Nacional de Córdoba. 
»Shady, R. y Leyva, C. (2003). La Ciudad Sagrada de Caral-Supe. Los Orígenes de la Civilización Andina y la Formación del Estado Prístino en el Antiguo Perú. Lima: PEACS/ INC.

» Singh, M., Kumar, S. V., Waghmare, S. A. y Sabale, P. D. (2016). Aragonite-vaterite-calcite: Polymorphs of $\mathrm{CaCO}_{3}$ in 7th century CE lime plasters of Alampur group of temples, India. Construction and Building Materials, 112, 386-397.

» Taylor, J. D., Kennedy, W. J. y Hall, A. (1969). The shell structure and mineralogy of the Bivalvia. Introduction. Nuculacae-Trigonacae. Bulletin of the British Museum (Natural History) Zoology, 3, 1-125.

» Téllez, F. (1997). Sthrophocheilus oblongus Müll: Restos de fauna malacológica tropical en contextos funerarios de San Pedro de Atacama: Norte de Chile (Informe preliminar). Estudios Atacameños, 14, 95-120.

» Weir, C. E. y Lippincott, E. R. (1961). Infrared studies of aragonite, calcite, and vaterite type structures in the borates, carbonates, and nitrates. Physics and Chemistry, $65 \mathrm{~A}(3)$, $173-183$. 